\title{
Nonisotropic Scattering Characteristic in an Alternant Tree-Blocked Viaduct Scenario on High-Speed Railway at $2.35 \mathrm{GHz}$
}

\author{
Rongchen Sun, ${ }^{1,2}$ Cheng Tao, ${ }^{1,2}$ Liu Liu, ${ }^{1,2}$ Zhenhui Tan, ${ }^{1}$ Lingwen Zhang, ${ }^{1}$ and Tao Zhou ${ }^{1}$ \\ ${ }^{1}$ Institute of Broadband Wireless Mobile Communications, School of Electronics and Information Engineering, \\ Beijing Jiaotong University, Beijing 100044, China \\ ${ }^{2}$ National Mobile Communications Research Laboratory, Southeast University, Nanjing 210096, China
}

Correspondence should be addressed to Rongchen Sun; rongchensun@gmail.com

Received 3 October 2013; Revised 9 March 2014; Accepted 23 March 2014; Published 26 June 2014

Academic Editor: Felipe Cátedra

Copyright (C) 2014 Rongchen Sun et al. This is an open access article distributed under the Creative Commons Attribution License, which permits unrestricted use, distribution, and reproduction in any medium, provided the original work is properly cited.

\begin{abstract}
This paper presents the nonisotropic scattering characteristic of the mobile radio channel in an alternant tree-blocked viaduct scenario on high-speed railway (HSR) by real field measuring at $2.35 \mathrm{GHz}$. An angle of arrival (AOA) probability density function (PDF) is proposed for the nonuniform AOA at the mobile caused by stochastically distributed scatterers. Two Von Mises angular distributions with broad applicability are used to represent the line of sight (LOS) component and part of the scattering component in the AOA model. Based on such a PDF statistical characteristics of Ricean factor, $\kappa$, and AOA of the scattering component are modeled in LOS and obstructed line of sight (OLOS) cases, respectively. The results may give a meaningful and accurate channel model and could be utilized in HSR viaduct scenario evaluation.
\end{abstract}

\section{Introduction}

HSR is becoming a major passenger transport tool in recent years due to its large transport capacity and high conveying efficiency. The speed of HSR in Japan, which is called Shinkansen, can reach up to $250 \mathrm{~km} / \mathrm{h}$ [1], and Eurostar [2] operates at $300 \mathrm{~km} / \mathrm{h}$ which is the same as the operating speed of China Railway High-speed (CRH) trains. It has brought a great convenience for the travel of passengers and has made a great contribution to the economic development of the cities along the railway. Recently China is planning to construct some new HSR lines, and the total mileage of China's HSR will reach $18.000 \mathrm{~km} \mathrm{[3].}$

With the rapid development of HSR, the corresponding communication systems are under construction to meet the basic needs for the passenger call services at the same time. However, it is obvious that the current communication quality and capacity are not beneficial enough to satisfy the increasing demands for data-rich services, such as Internet access, video call service, and streaming media service. When the train speed reaches $200 \mathrm{~km} / \mathrm{h}$, the fading characteristics of the wireless channel can be extremely changed compared with that in typical scenarios. As a result, large Doppler shift makes the channel state vary a lot, and the Doppler spread can lead to intercarrier interfaces in multicarrier systems [4]. Both of these influences can reduce the performance of the communication systems. Therefore Doppler is a critical factor of HSR scenarios communication simulation. Doppler spectra of the received signal depend on the AOA PDF of multipath signals reflected from the scatterers in the propagation environment. The classic Doppler spectrum using isotropic scattering assumption is generally not suitable for most of the scenarios in reality. Considering the propagation channel in HSR scenarios, it can be divided into several scenarios such as viaduct, $\mathrm{U}$-shape groove, and tunnel and all of these have their own propagation characteristics due to the nonisotropic nature of the environment. Among these scenarios, viaduct is a common one which appears frequently along the whole HSR line. Large and small scale characteristics in a viaduct scenario on the Beijing-Tianjin HSR line 
have been investigated in [5] and the rapid Doppler transition demonstrated in [6] is verified. Some valuable results of statistical angle information and Doppler spectra versus time are obtained in [7] without distinction between the different scenarios explicitly. Additionally, the receiver inside the train can also receive the scattering signals reflected by the carriage during the measurement. However, in these papers a deterministic AOA PDF is not given and furthermore the closed form correlation function and Doppler spectrum are not deduced. Three Doppler spectra and AOA distribution for highway scenario are compared in [8], but they are not flexible enough to describe the environment with different scatterer densities. A two-parameter Von Mises distribution is used to estimate the AOA PDF in nonline of sight (NLOS) environment [9], in which the measurement data have only the scattering component in line with a zero-mean complex Gaussian process. Meanwhile, the relevant correlation function and Doppler model are given. However, estimated and modeling results with both LOS and scattering component in strong LOS environment, such as HSR scenarios, have not been studied in any of the published literature.

The main contribution of this paper is to firstly establish the multipath fading model of an alternant tree-blocked viaduct scenario based on the experimental data. Meanwhile, by using the Von Mises distribution, the statistical models of the Ricean factor, AOA, are also extracted in this specific environment. These parameters are helpful to determine the correlation function and Doppler characteristics and furthermore give advice to communication system design.

The remainder of this paper is organized as follows. In Section 2, a brief account of the measurements campaign and equipment is given. Then, a modified parametric multipath fading model is provided in Section 3. Parameters estimation and statistical results are exhibited in Section 4. Finally, conclusions are drawn in the final section.

\section{Measurement Campaign and Equipment}

The measurement is carried out on Zhengzhou-Xi'an (ZX) HSR, using the Elektrobit Propsound Channel Sounder. The transmitter continuously generates a pseudonoise (PN) sequence as the probe signal. At the receiver side, the channel impulse responses (CIRs) are estimated by slide correlating the received signal with a sequence identical to the one used at the transmitter. The clocks of the transmitter and the receiver were synchronized by the global positioning system (GPS) network which provides a very accurate universal timing signal to all GPS receivers. The GPS network also can be used for distance determination. The measurement configuration is listed in Table 1.

The viaduct scenario is near the north of Huashan Station with a length of $1.6 \mathrm{~km}$. The height of the viaduct is about $8 \mathrm{~m}$. The transmitter antenna, a vertical polarized dipole as shown in Figure 2(a), is placed on the top of a roof and the height is $12.8 \mathrm{~m}$ above the ground. The receiver antenna, a wideband vertical-polarized antenna HUBER + SUHNER [10] as shown in Figure 2(b), is positioned in the middle of the train, mounted on the top with the height of $3 \mathrm{~m}$ above
TABLE 1: Measurement parameters.

\begin{tabular}{lc}
\hline Parameter & Value \\
\hline Carrier frequency & $2.35 \mathrm{GHz}$ \\
Bandwidth & $50 \mathrm{MHz}$ \\
Transmit power & $30.8 \mathrm{dBm}$ \\
PN length & 127 \\
Channel sample rate & $1968.5 \mathrm{~Hz}$ \\
\hline
\end{tabular}

the track. The train moves at a velocity of $200 \mathrm{~km} / \mathrm{h}$. Figure 1 shows that there are couples of trees alongside the viaduct. Some of them are as tall as the train and some of them are not. Therefore the whole measurement environment is divided into LOS and OLOS segments by the trees with different density and height, and the parameters will be analyzed separately. Distance between $416 \mathrm{~m}$ and $607 \mathrm{~m}$ is the LOS segments, and other areas belong to the OLOS segment. In Figure $1(\mathrm{a}) d_{\min }$ is the perpendicular distance from the transmitter to the viaduct, and FP is short for foot point. $d$ is the distance between the receiver and the point FP. Due to the symmetrical characteristic of the environment, the data segment before the train running across FP was selected in the analysis process.

\section{A Parametric Multipath Fading Model}

As mentioned above, the sparse scatterers alongside the railway result in the nonisotropic feature of the distribution of AOA. This characteristic gives rise to some particular correlation functions and Doppler spectra differ from the Bessel function and U-shape power spectrum in Clarke's two-dimensional scattering model based on the isotropic scattering assumption; that is, the AOA follows a uniform distribution over $[-\pi, \pi)$. Various angular PDFs are proposed in the literature published, such as Laplace PDF [11], cosine PDF [12], and AOA PDF of Jakes X, round, and flat Doppler spectra model in [8], and all of them are used to describe the nonisotropic scattering property to a certain point. However, those PDFs are not flexible enough to fit the changeable environment, which reflects a random directional characterization of the scatterers.

Generally, Von Mises distribution is used for the directional distribution of the angle of scattering, and the PDF $p_{\mathrm{Von}}(\theta)$ is given by

$$
p_{\mathrm{Von}}(\theta)=\frac{1}{2 \pi I_{0}(\kappa)} e^{\kappa \cos (\theta-\alpha)}, \quad \theta \in(-\pi, \pi],
$$

where $(\kappa, \alpha)$ are the two parameters that completely control $p_{\mathrm{Von}}(\theta) ; \alpha \in[-\pi, \pi)$ and $\kappa$ denote the mean angle and spread of AOA of waves from scatterers and $I_{0}(\cdot)$ is the zero-order modified Bessel function. As can be deduced from (1), in the extreme cases for $\kappa=0, p_{\text {Von }}(\theta)=1 / 2 \pi$ can be obtained which result in a classic U-shape spectra, while for $\kappa=\infty$ we get $p_{\mathrm{Von}}(\theta)=\delta(\theta-\alpha)$, which can be used to represent the AOA $\theta_{\mathrm{LOS}}$ of the LOS path because $\theta_{\mathrm{LOS}}$ is equivalent to $\theta_{\mathrm{LOS}} \cdot \delta\left(\theta-\theta_{\mathrm{LOS}}\right)$, where $\delta(\cdot)$ is the Dirac delta function. 


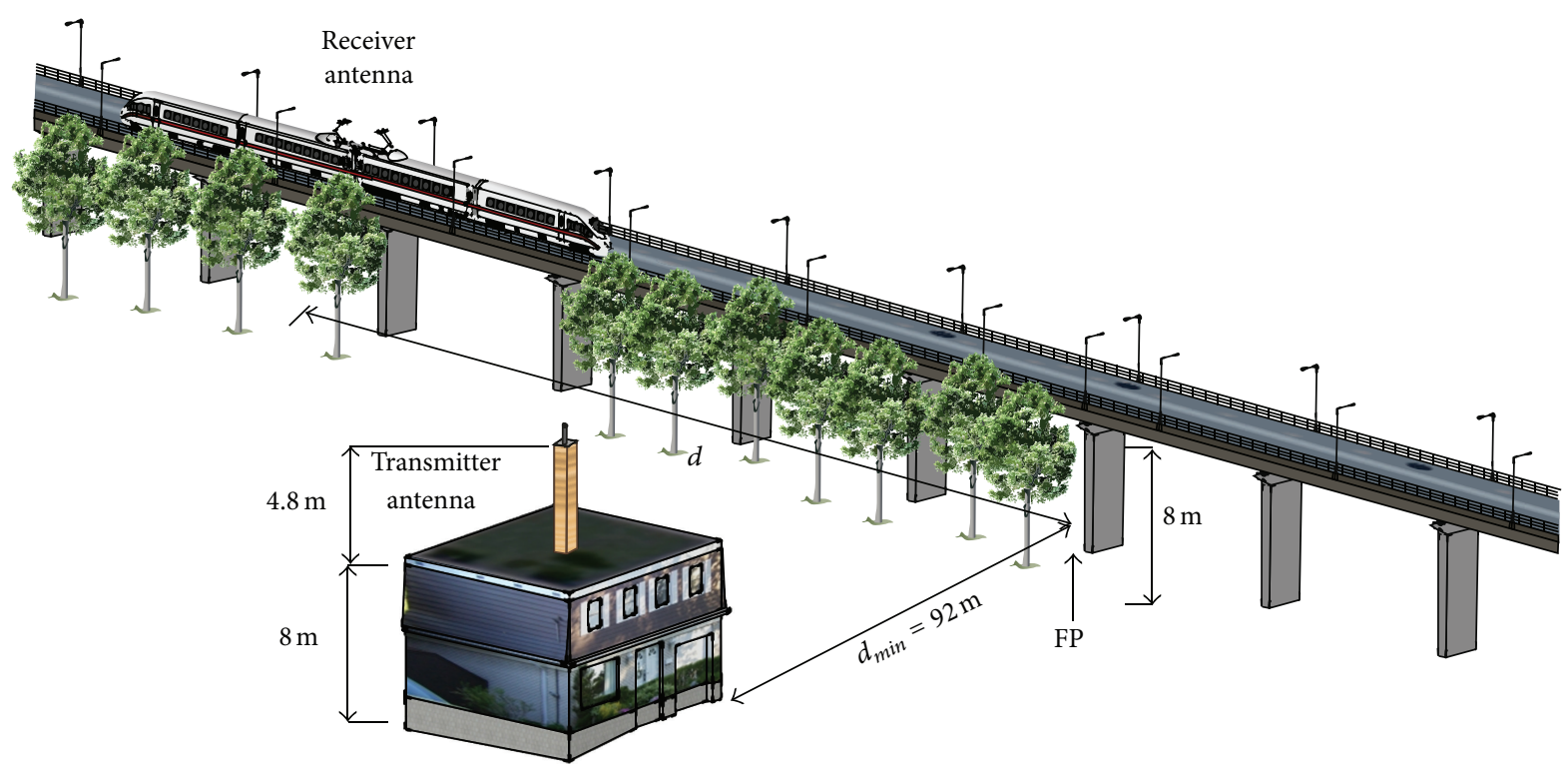

(a)

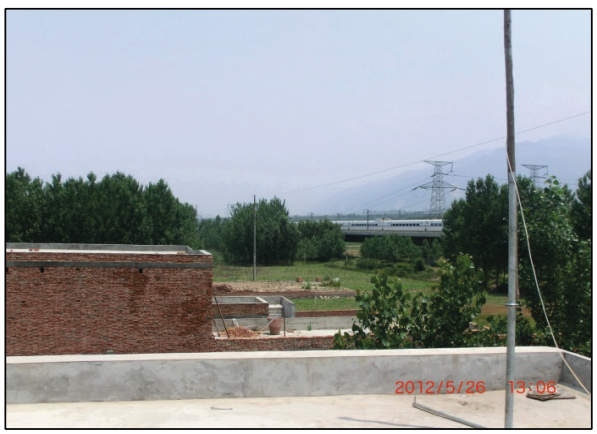

(b)

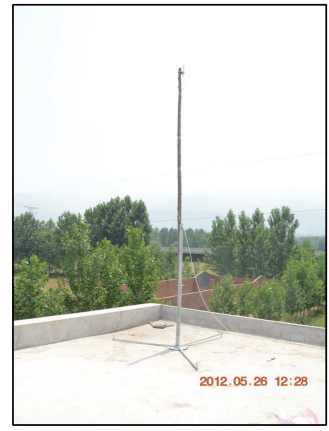

(c)

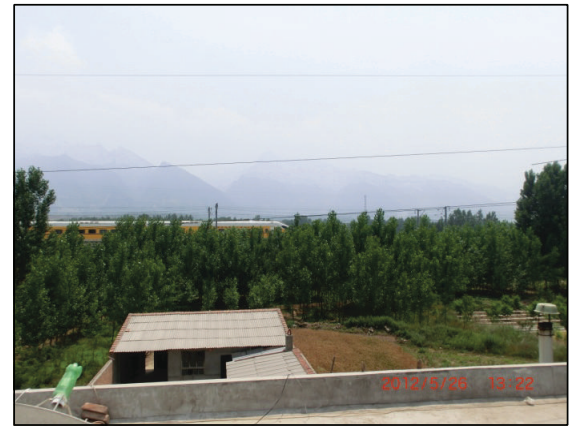

(d)

FIGURE 1: Propagation environment: (a) measurement scenario, (b) and (d) photos of the propagation region remote and close to the transmitter, and (c) transmitter antenna.

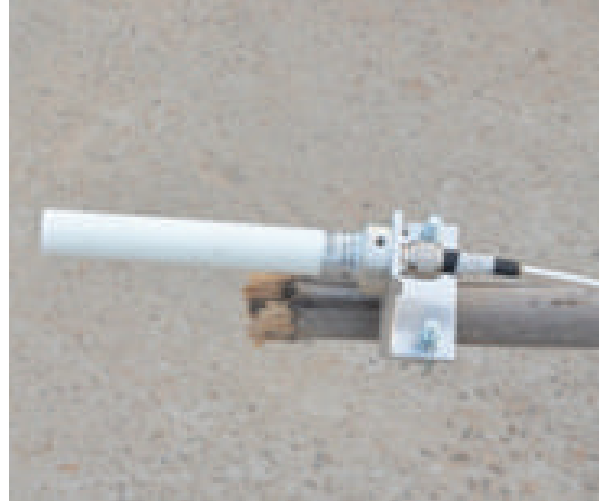

(a) Vertical polarized dipole

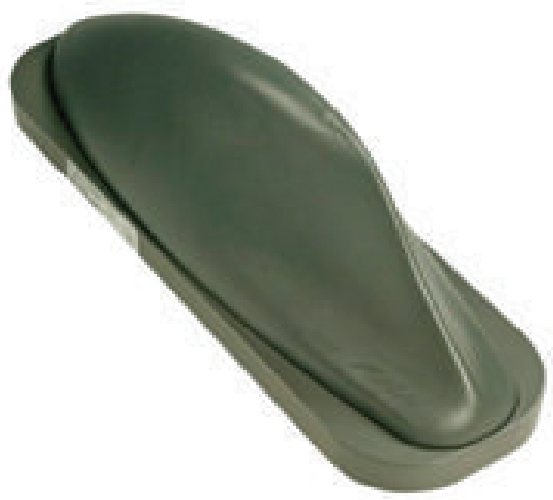

(b) HUBER + SUHNER

Figure 2: The features of antennas used at (a) TX and (b) RX. 
The multipath component $h(t)$ can be expressed by the sum of exponential function, and it is given by

$$
\begin{aligned}
h(t)= & \frac{1}{\sqrt{K+1}} \lim _{N \rightarrow \infty} \sum_{n=1}^{N} c_{n} e^{j\left(2 \pi f_{\max } \cos \left(\theta_{n}\right) t+\phi_{n}\right)} \\
& +\sqrt{\frac{K}{K+1}} e^{j\left(2 \pi f_{\max } \cos \left(\theta_{L O S}\right) t+\phi_{L O S}\right)},
\end{aligned}
$$

where $K$ is the Ricean factor; $N$ is the number of the scatterers; $j=\sqrt{-1}$ and the coefficient $c_{n} \in[0,1)$ satisfies $\lim _{N \rightarrow \infty} \sum_{n=1}^{N} c_{n}^{2}=1$ in order to normalize the power of the multipath $E|h(t)|^{2}$ to one; $f_{\max }$ is the maximum Doppler frequency in $\mathrm{Hz}$ which is equal to $v f_{c} / c$, and $v$ is the moving velocity of the receiver, $f_{c}$ is the carrier frequency, $c$ is the speed of light with a value of $3 \times 10^{8} \mathrm{~m} / \mathrm{s} ; \theta_{n}, n=1, \ldots, N$ is the AOA of the $n$th incoming echo, and all of $\theta_{n}$ are independent and identically distributed (i.i.d.) with a distribution $p_{\text {scat }}(\theta)$; phases $\phi_{n}$ are i.i.d. with a uniform distribution over $[-\pi, \pi)$; $\theta_{\mathrm{LOS}}$ is the AOA of the LOS component relative to the moving direction and $\phi_{\mathrm{LOS}}$ is the relevant phase. As is well known, the correlation function of $h(t)$ can be given by

$$
\begin{aligned}
\phi_{h h}(\tau) & =E\left[h^{*}(t) h(t+\tau)\right] \\
& =\int_{-\pi}^{\pi} p(\theta) e^{j 2 \pi f_{\max } \cos \pi(\theta) \tau} d \theta,
\end{aligned}
$$

where $p(\theta)$ contains the distribution of the scattering component as well as the LOS component and $\theta$ is the AOA from all the received multipath components. In the literature [9], it has already been discussed that the accuracy using a distribution combined with both isotropic and nonisotropic components is better than that using nonisotropic distribution alone in fitting the measurement data. Thus in theory $p(\theta)$ can be written as

$$
\begin{gathered}
p(\theta)=\frac{1}{K+1} p_{\text {scat }}(\theta)+\frac{K}{K+1} p_{\text {LOS }}(\theta) \\
p_{\text {scat }}(\theta)=\eta \frac{1}{2 \pi I_{0}\left(\kappa_{\text {scat }}\right)} e^{\kappa_{\text {scat }} \cos \left(\theta-\theta_{\text {scat }}\right)}+(1-\eta) \frac{1}{2 \pi} \\
p_{\text {LOS }}(\theta)=\delta\left(\theta-\theta_{\text {LOS }}\right) .
\end{gathered}
$$

However, in practical terms the angle spread of the LOS component is not negligible. This result in that $\delta(\cdot)$ function is not capable entirely of accurate parameter estimation. For this reason another Von Mises distribution with $\kappa \geq 50$ was adopted instead of fitting the LOS component in the measurement data and (4) can be expanded as

$$
\begin{aligned}
p(\theta)= & \frac{1}{K+1}\left(\eta \frac{1}{2 \pi I_{0}\left(\kappa_{\text {scat }}\right)} e^{\kappa_{\text {scat }} \cos \left(\theta-\theta_{\text {scat }}\right)}+(1-\eta) \frac{1}{2 \pi}\right) \\
& +\frac{K}{K+1} \frac{1}{2 \pi I_{0}\left(\kappa_{\text {LOS }}\right)} e^{\kappa_{\mathrm{LOS}} \cos \left(\theta-\theta_{\mathrm{LOS}}\right)},
\end{aligned}
$$

where $\left(\kappa_{\text {scat }}, \theta_{\text {scat }}\right)$ and $\left(\kappa_{\mathrm{LOS}}, \theta_{\mathrm{LOS}}\right)$ are the parameters of Von Mises distribution mentioned previously for scattering and
LOS component, respectively; $\eta \in[0,1]$ is a coefficient which represents the amount of nonisotropic scattering component.

Substituting (7) into (3), the correlation function $\phi_{h h}(\tau)$ can be given by

$$
\begin{aligned}
\phi_{h h}(\tau)= & \frac{1}{K+1}\left(\eta \phi_{h h}^{\mathrm{scat}}(\tau)+(1-\eta) J_{0}\left(2 \pi f_{\max } \tau\right)\right) \\
& +\frac{K}{K+1} \phi_{h h}^{\mathrm{LOS}} .
\end{aligned}
$$

By using (1) in (3), $\phi_{h h}^{X}(\tau)$ can be written as

$$
\phi_{h h}^{X}(\tau)=\frac{I_{0}\left(\sqrt{\kappa_{X}^{2}-4 \pi^{2} f_{\max }^{2} \tau^{2}+j 4 \pi \kappa_{X} \cos \left(\theta_{X}\right) f_{\max } \tau}\right)}{I_{0}\left(\kappa_{X}\right)},
$$

where $\phi_{h h}^{X}(\tau)$ is the correlation function of received signal with the AOA following a Von Mises distribution. It represents the correlation functions of nonisotropic part in scattering component and LOS component when the superscript $X$ is scat and LOS, respectively.

The Doppler spectrum $S_{h h}(f)$ is the Fourier transform of $\phi_{h h}(\tau)$. According to [9], $S_{h h}(f)$ can be written as

$$
\begin{aligned}
& S_{h h}(f)= \frac{1}{K+1}\left(\eta S_{h h}^{\text {scat }}(f)+(1-\eta) S_{h h}^{\text {classic }}(f)\right) \\
&+\frac{K}{K+1} S_{h h}^{\mathrm{LOS}}(f), \\
& S_{h h}^{X}(f)=\frac{e^{\kappa_{X} \cos (\alpha)\left(f / f_{\max }\right)} \cosh \left[\kappa_{X} \sin \left(\theta_{X}\right) \sqrt{1-\left(f / f_{\max }\right)^{2}}\right]}{\pi I_{0}\left(\kappa_{X}\right) \sqrt{f_{\text {max }}^{2}-f^{2}}}, \\
& \quad|f| \leq f_{m},
\end{aligned}
$$

where $S_{h h}^{X}(f)$ is the Fourier transform of $\phi_{h h}^{X}(\tau)$ and

$$
S_{h h}^{\text {classic }}(f)=\frac{1}{\pi \sqrt{f_{\max }^{2}-f^{2}}}, \quad|f| \leq f_{m}
$$

is the Jakes Doppler spectrum.

The estimation method we used here is nonlinear least squares method [13]:

$$
\begin{aligned}
& \left(\widehat{K}, \widehat{\kappa}_{\text {LOS }}, \widehat{\theta}_{\text {LOS }}, \widehat{\kappa}_{\text {scat }}, \widehat{\theta}_{\text {scat }}, \widehat{\eta}\right) \\
& \quad=\arg \min \left(\frac{1}{n} \sum_{l=1}^{n}\left[\widehat{\phi}_{h h}\left(\tau_{l}\right)-\phi_{h h}\left(\tau_{l}\right)\right]^{2}\right) .
\end{aligned}
$$

And the correlation function of $h(t)$ was chosen as the target function in the estimation. $h(t)$ is the combined result in the delay domain of CIRs $h(t, \tau)$ which the channel sounder gives and the combination method can be expressed as

$$
h(t)=\int h(t, \tau) d \tau
$$




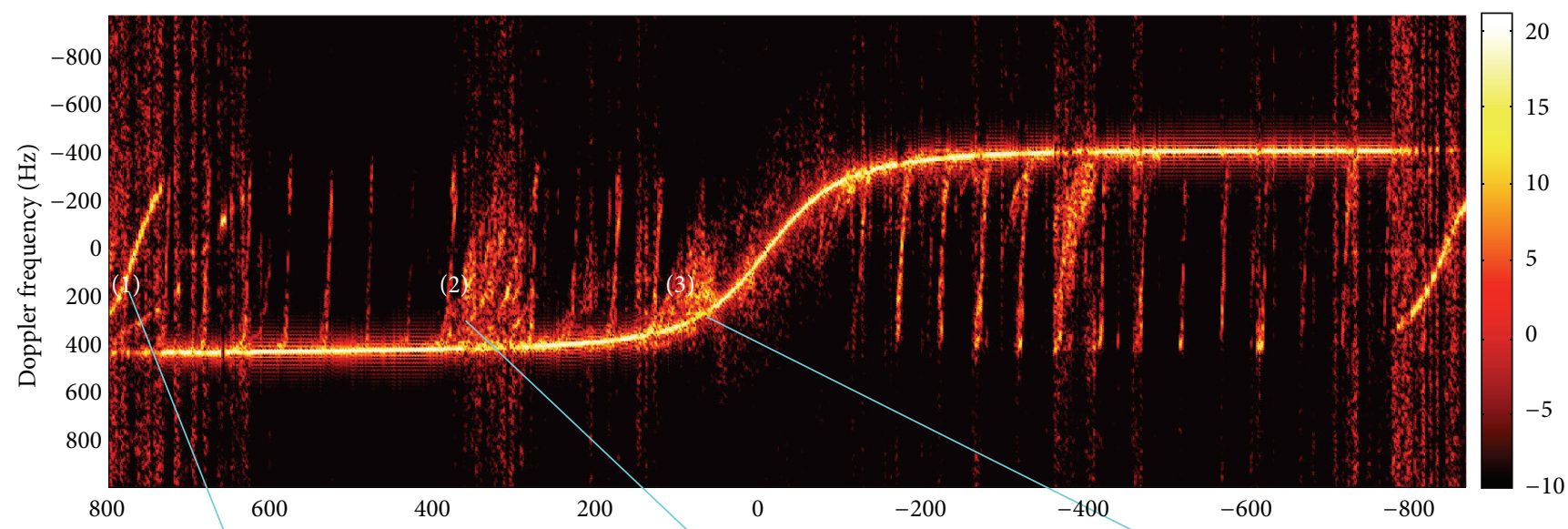

(a) Distance between the train and FP (m)

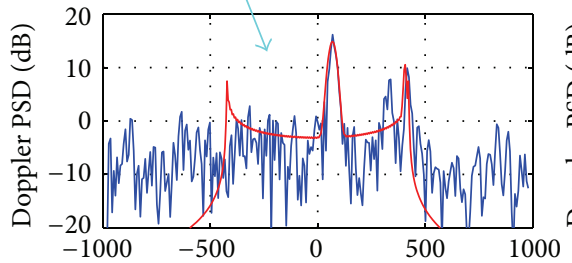

(b) Doppler frequency $(\mathrm{Hz})$

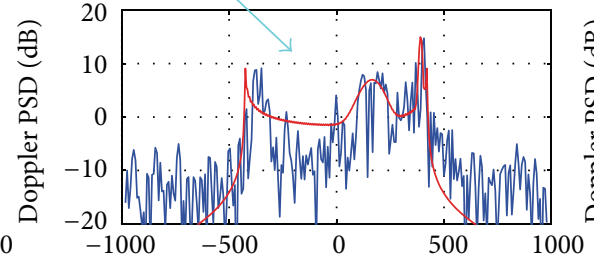

(c) Doppler frequency $(\mathrm{Hz})$

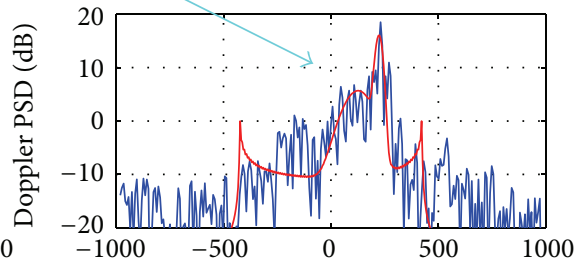

(d) Doppler frequency $(\mathrm{Hz})$

FIGURE 3: Doppler spectra characteristics in viaduct scenario.

\section{Measurement Results and Parameters Estimation}

As described in Section 2, we get about 60000 CIRs with a channel sampling rate of $1968 \mathrm{~Hz}$, which corresponds to $1.6 \mathrm{~km}$ in distance. Due to the symmetry of the environment, shown in Figure 3(a), we select the data before the train runs across the transmitter. 29000 CIRs and $n=128$ are used here and the estimated results are shown in the figures.

4.1. Doppler Transition and Doppler PSD (Power Spectrum Density) Estimated Results. Figure 3(a) illustrates the Doppler spectra during the whole measurement process, which is almost symmetric about the FP. Doppler spectra here is derived from the Fourier transform of the normalized correlation function. The regular highlight lines with an interval of about $50 \mathrm{~m}$ are some local Doppler transitions caused by the reflection of electric line pole since the antenna we used is vertically polarized. Scattering from trees and high-voltage line towers are also clear in the figure. For instance, Figures 3(b), 3(c), and 3(d) are the Doppler PSDs at $775 \mathrm{~m}, 370 \mathrm{~m}$, and $65 \mathrm{~m}$ from the FP. The blue and red lines are the Doppler PSDs of the measurement data and proposed model, respectively. Good fit can be seen between the two Doppler PSDs. Although the objective function is the correlation function in the estimation, Doppler PSD is more intuitive to observe the estimation accuracy. The estimated parameters at the three positions can be seen in Table 2 .

4.2. Ricean Factor. Firstly Figure 4 is the Ricean factor versus distance obtained by utilizing the approach in Section 3.
TABLE 2: Estimated parameters at the three positions.

\begin{tabular}{lcccccc}
\hline Position & $\widehat{K}(\mathrm{~dB})$ & $\widehat{\kappa}_{\text {LOS }}$ & $\widehat{\theta}_{\text {LOS }}\left(^{\circ}\right)$ & $\widehat{\kappa}_{\text {scat }}$ & $\widehat{\theta}_{\text {scat }}\left({ }^{\circ}\right)$ & $\widehat{\eta}$ \\
\hline 1 & -11.84 & 628.51 & 16.92 & 700 & 80.70 & 0.65 \\
2 & -5.10 & 700 & 21.87 & 65.65 & 67.11 & 0.35 \\
3 & 3.47 & 700 & 58.18 & 54.71 & 72.91 & 0.81 \\
\hline
\end{tabular}

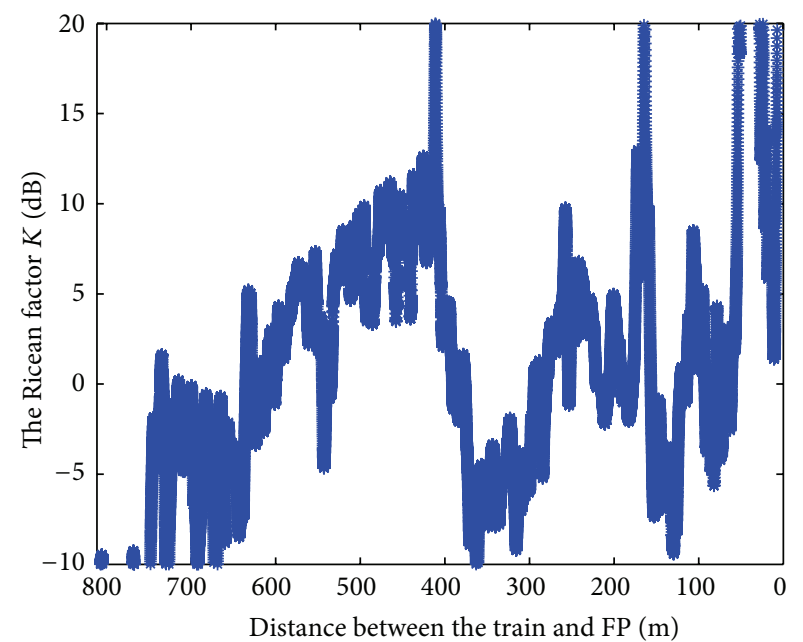

FIGURE 4: Ricean factor versus the distance.

The reference point FP is the initial position which means $d=0$. The same definition is used in all the figures. Based on the two segments defined in Section 2, Ricean factor in LOS and OLOS segments is modeled as a linear model varying 


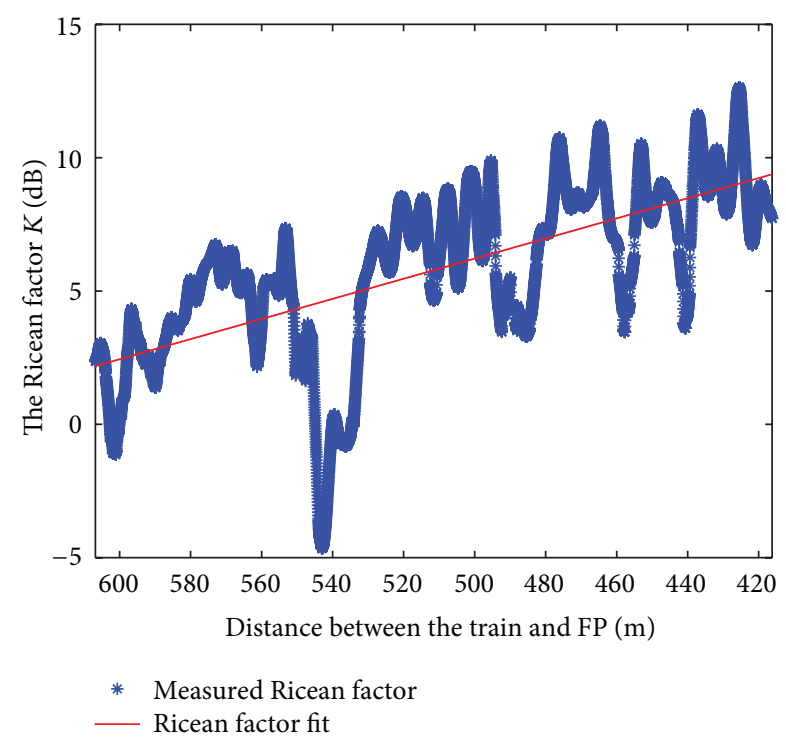

FIgURE 5: Ricean factor model in LOS segment.

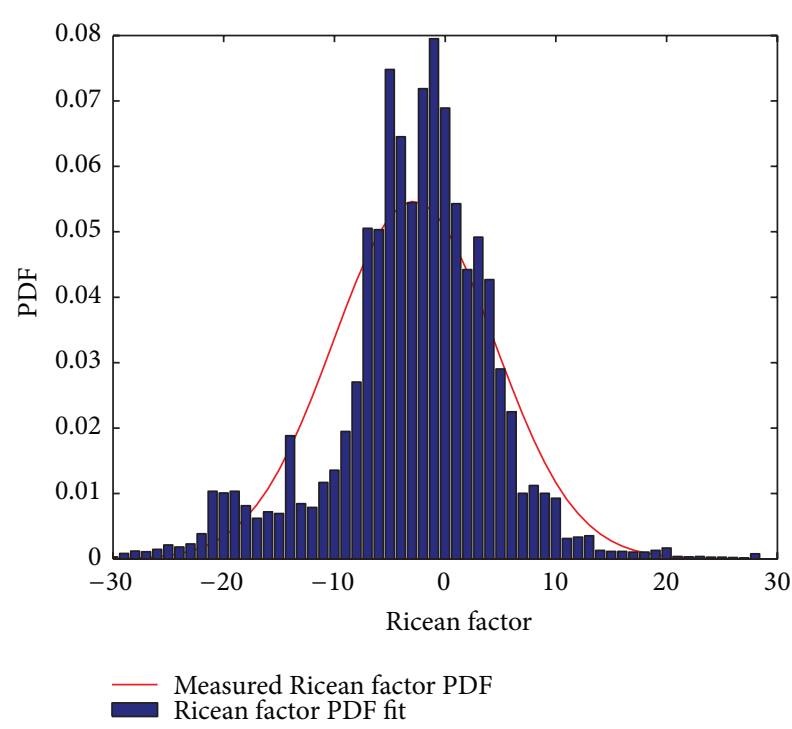

FIGURE 6: Statistical characteristic of Ricean factor in OLOS segment.

with distance and a Gaussian variable, respectively. Ricean factor model in LOS segment is depicted in Figure 5 and the red fitting curve is expressed as

$$
K_{\mathrm{LOS}}=-0.038 \cdot d+25.09 \text {. }
$$

Figure 6 gives the statistical characteristic of Ricean factor in OLOS segment. In this figure, the blue bars represent the PDF of the measured Ricean factor and the red curve is the normal fitting PDF whose mean value is -2.8 and standard deviation is 7.3. It is noted that the standard deviation in OLOS segment is noticeable to be big for a single scenario. The main reason for this phenomenon is that heights of the alternant trees alongside the viaduct vary a lot. This gives rise to a scenario composed of LOS, OLOS, and NLOS

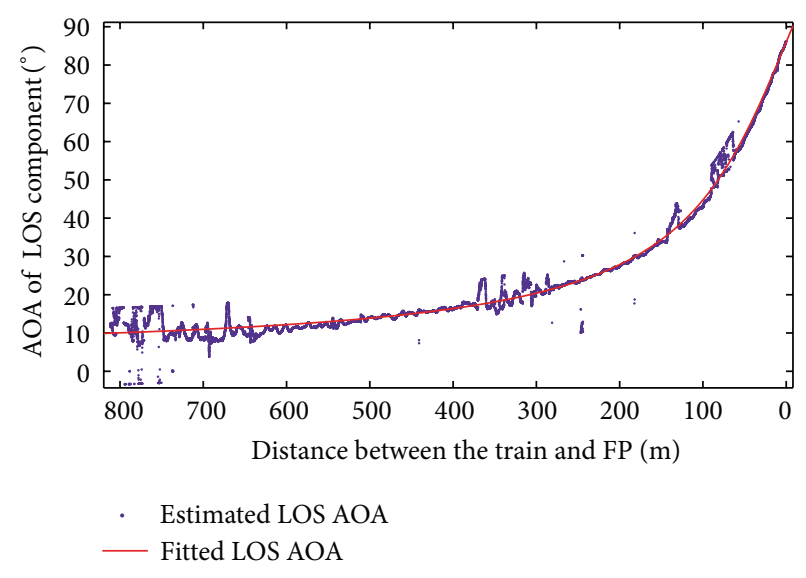

FIGURE 7: Estimated and fitting results of $\theta_{\text {LOS }}$.

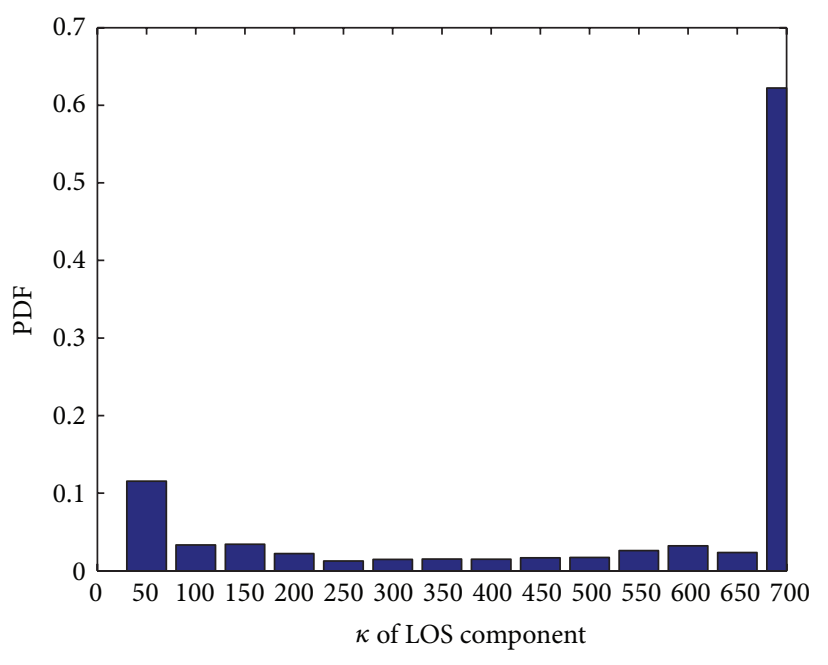

FIGURE 8: PDF of $\widehat{\kappa}_{\text {LOS }}$.

occasionally, which cannot be distinguished clearly. As a result, the Ricean factor varies widely around the mean value.

4.3. Estimated Results of $\left(\widehat{\kappa}_{\text {LOS }}, \widehat{\theta}_{\text {LOS }}\right)$. Secondly, Figures 7 and 8 illustrate two desirable outcomes of $\left(\widehat{\kappa}_{\text {LOS }}, \widehat{\theta}_{\text {LOS }}\right)$ which were extracted from the measurement data. A theoretic model of $\theta_{\text {LOS }}$ on railway condition is given in [6]. We fitted the estimated results using the theoretic model and then an acceptable error was gotten as

$$
\widehat{\theta}_{\mathrm{LOS}}=0.917 \cdot \theta_{\text {theretic }}+3.5 \text {. }
$$

The curve of $\widehat{\theta}_{\text {LOS }}$ was plotted with redline in Figure 7. As the figure shows, the curve overlaps the estimated value perfectly. Figure 8 exhibits a PDF of $\widehat{\kappa}_{\text {LOS }}$, and, as expected, most of the values are extremely large, for instance, near to 700 , to make the second term in (7) represents the LOS component accurately.

4.4. Estimated and Modeling Results of $\left(\widehat{\kappa}_{\text {scat }}, \widehat{\theta}_{\text {scat }}\right)$. Estimated results of $\left(\widehat{\kappa}_{\mathrm{LOS}}, \widehat{\theta}_{\mathrm{LOS}}\right)$ displayed above make more sense 


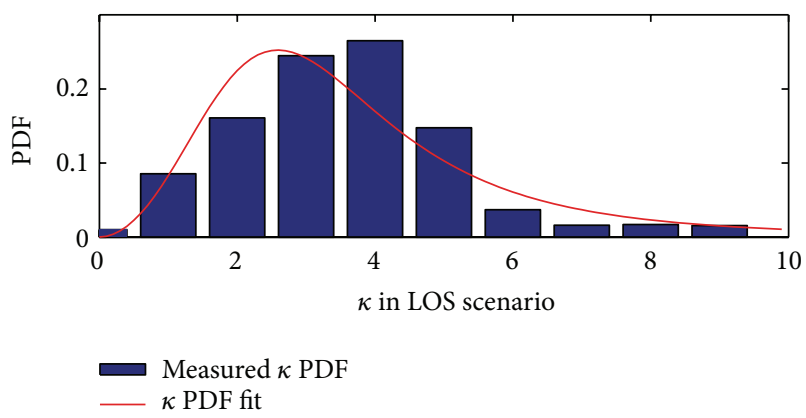

(a)

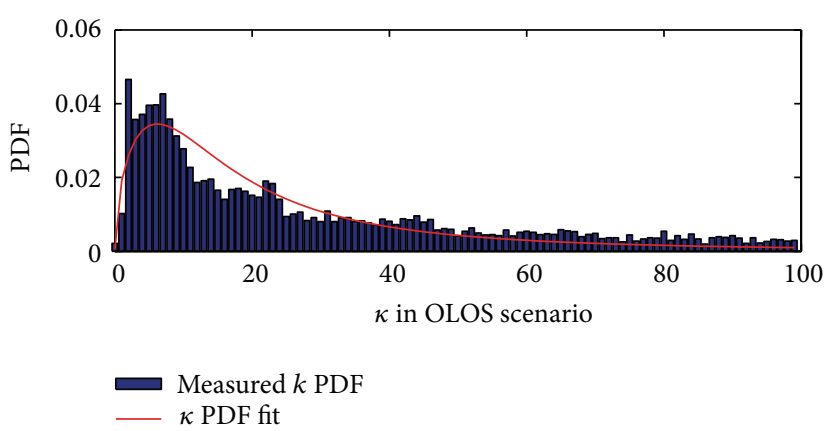

(b)

FIGURE 9: Statistical characteristic of $\widehat{\kappa}_{\text {scat }}$ in LOS and OLOS segment.

TABLE 3: Statistical distribution of $\widehat{\kappa}_{\text {scat }}$.

\begin{tabular}{lcc}
\hline Item & Mean value & Standard deviation \\
\hline$\widehat{\kappa}_{\text {scat }}$ in LOS & 1.19 & 0.34 \\
$\widehat{\kappa}_{\text {scat }}$ in OLOS & 2.87 & 0.66 \\
\hline
\end{tabular}

in being an accurate verification than being a conclusion. The main contribution in this paper is the modeling of the statistical and analytical results on $\left(\widehat{\kappa}_{\text {scat }}, \widehat{\theta}_{\text {scat }}\right)$ of the scattering component.

We consider discussing the PDF of $\left(\widehat{\kappa}_{\text {scat }}, \widehat{\theta}_{\text {scat }}\right)$ in LOS and OLOS segments separately. Figure 9 shows statistical characteristic of $\widehat{\kappa}_{\text {scat }}$ in LOS and OLOS segment. The red lines are the curves fitted by the log-logistic distribution, whose $\mathrm{PDF}$ is given by (17). The mean value $\mu$ and standard deviation $\sigma$ in each segment are listed in Table 3. Consider

$$
y=\frac{1}{\sigma x} \cdot \frac{e^{(\ln x-\ln \mu) / \sigma}}{\left(1+e^{(\ln x-\ln \mu) / \sigma}\right)^{2}} .
$$

By comparing these parameters in the three figures, it can be found that $\widehat{\kappa}_{\text {scat }}$ in LOS segment is apparently larger than $\widehat{\kappa}_{\text {scat }}$ in OLOS segment. Most of $\widehat{\kappa}_{\text {scat }}$ range from 1 to 6 with the value between 3 and 5 accounting for nearly $50 \%$ in LOS segment. In OLOS segment the majority of $\widehat{\kappa}_{\text {scat }}$ centred between 0 and 50 , and the number of the value greater than 50 is extremely small. The main reason is the difference between scattering environment of the two segments. From an intuitive perspective, in LOS segment the scattering component is so much lower than the LOS component resulting in the low extent of the nonisotropic scattering. Nevertheless, with the Ricean factor decreasing, the nonisotropic scattering component becomes more apparent compared to the LOS component and meanwhile $\widehat{\kappa}_{\text {scat }}$ gets larger.

Figure 10 depicts the value of $\widehat{\theta}_{\text {scat }}$ versus distance. It is clear to see the boundary of the LOS and OLOS segments. As Figure 11 shows, in LOS segment most of $\widehat{\theta}_{\text {scat }}$ center around $\theta_{\mathrm{LOS}}$. And, after checking out the measurement environment, some local scattering trees near the transmitter side are considered as the reason causing the spread of the AOA from the direction of the transmitter. Therefore $\widehat{\theta}_{\text {scat }}$ in LOS

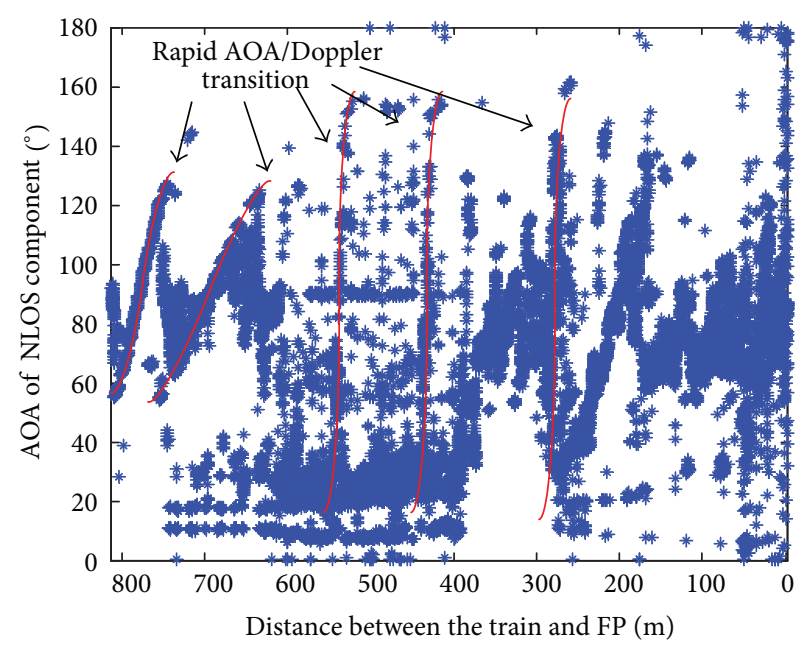

FIGURE 10: Value of $\widehat{\theta}_{\text {scat }}$ versus distance.

TABLE 4: Statistical distribution of $\widehat{\theta}_{\text {scat }}$.

\begin{tabular}{lcc}
\hline Item & Mean value & Standard deviation \\
\hline AOA in LOS & 27.12 & 9.16 \\
AOA in OLOS & 75.81 & 22.20 \\
\hline
\end{tabular}

segment are assumed to follow the normal distribution with the mean values $\mu=27.12$ and $\sigma=9.16$ (shown in Table 4).

Obviously, unlike the LOS segment, $\widehat{\theta}_{\text {scat }}$ vary around 75.81 degrees with a standard deviation of $\sigma=22.20$ fitted by a normal distribution in OLOS segment (shown in Table 4). This distribution characterization is probably caused by the scattering echoes from the trees and other scatterers like high-voltage line towers and electric line pole along the track on the viaduct. When the high speed train runs across a certain scatterer, according to Huygens principle, the scatterers become new signal sources; therefore the AOA and Doppler shift of the scattering component in the receiving signals vary from positive to negative over a period of time related to the distance between the train and the scatterers (red line in Figure 10). In the meanwhile, $\widehat{\theta}_{\text {scat }}$ change in the same trend centered on 90 degrees approximately. 


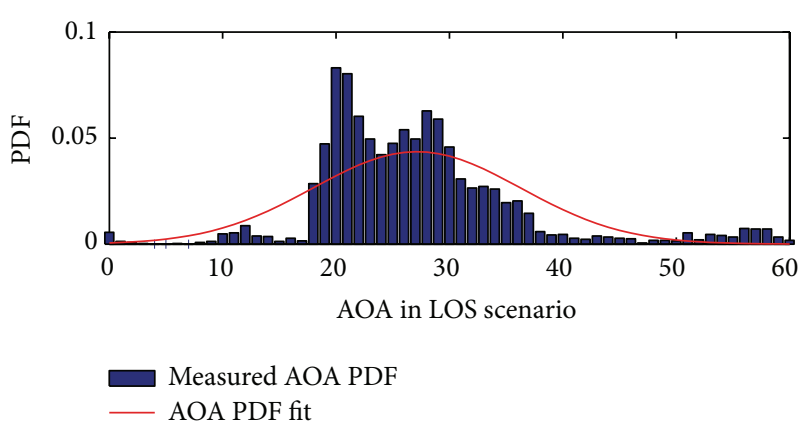

(a)

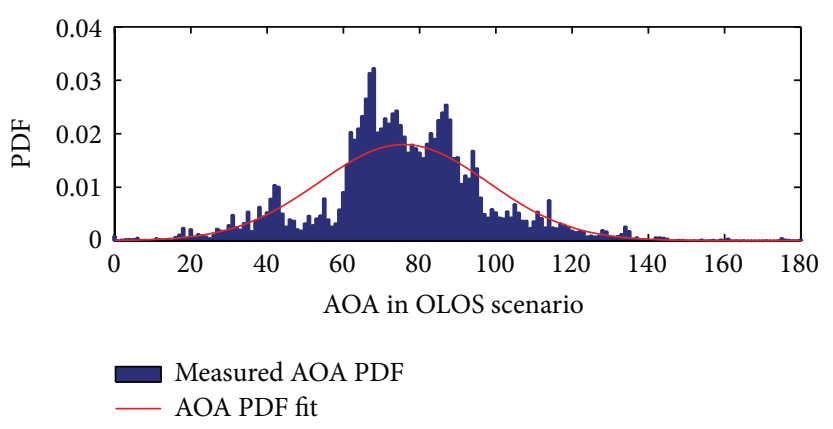

(b)

FIGURE 11: Statistical characteristic of $\widehat{\theta}_{\text {scat }}$ in LOS and OLOS segment.

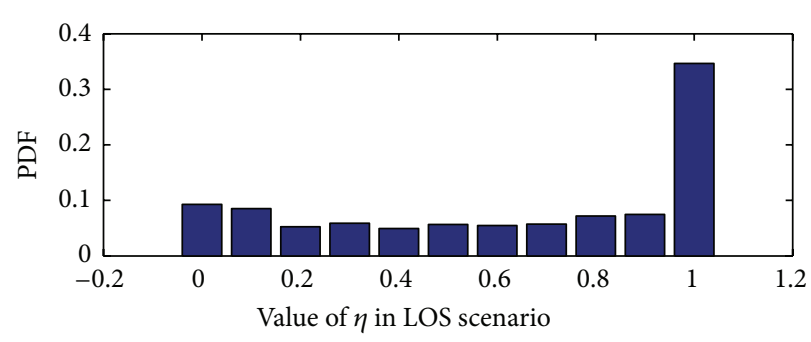

(a)

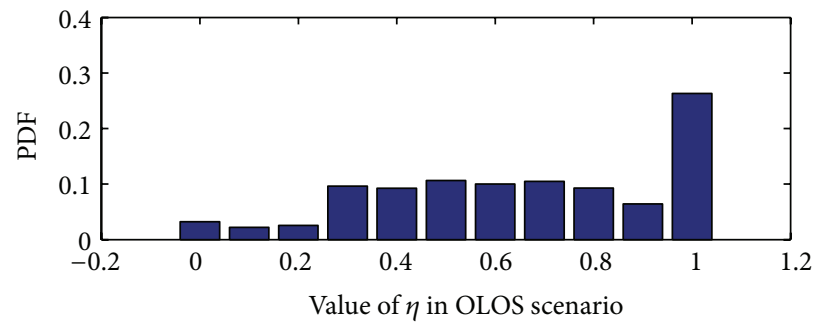

(b)

FIGURE 12: PDF of $\eta$ in LOS and OLOS section.

4.5. Statistic Characteristic of $\eta$. The statistical PDF results are depicted in Figure 12. The PDF shows two different parts apparently. Thus, $\eta$ can be modeled as two piecewise parts over $\eta \in(0,1)$ and $\eta=1$, respectively. The modeling results of $\eta$ are listed in Table 5. The value of $\eta$ in $\eta \in(0,1)$ follows a uniform distribution. The reason why $\eta=1$ accounts more than other values is that moderate correlation between $\eta$ and $\widehat{\kappa}_{\text {scat }}$, which is about -0.6 , was found during the analysis. Due to the sparse scatterers around the HSR, especially in the viaduct section, the signal reflected from scatterers is much lower than the LOS path, and most of the $\widehat{\kappa}_{\text {scat }}$ are small to fit the scattering component. In this case the isotropic component in (5) does not work anymore, since the Von Mises distribution with small $\kappa$ is more suitable than the uniform distribution in fitting the scattering component in the measurement data which is not ideal isotropic. And it can be also deduced that the probability of $\eta=1$ in LOS segment is a little bigger than that in OLOS segment according to the results in Section 4.4.

\section{Conclusion}

In this paper we proposed a modified AOA distribution model of the received multipath signals, which combines Von Mises and uniform distribution. This model was used to fit the data obtained from a measurement campaign at $2.35 \mathrm{GHz}$ employing a commercial channel sounder on ZX HSR line. Six parameters versus distance are estimated using the correlation function of combined CIR $h(t)$ as the target
TABLE 5: Statistical probability of $\eta$.

\begin{tabular}{lcc}
\hline Item & Probability of $\eta \in(0,1)$ & Probability of $\eta=1$ \\
\hline$\eta$ in LOS & 0.70 & 0.30 \\
$\eta$ in OLOS & 0.78 & 0.22 \\
\hline
\end{tabular}

function. According to the density of scatterers, the measurement environment is divided into LOS and OLOS segments. Ricean $K$ factor is modeled as a variable varying linearly with distance and a Gaussian variable separately based on the two segments defined. $\left(\widehat{\kappa}_{\mathrm{LOS}}, \widehat{\theta}_{\mathrm{LOS}}\right)$ are obtained for estimation accuracy validation purposes. Then $\left(\widehat{\kappa}_{\text {scat }}, \widehat{\theta}_{\text {scat }}\right)$ in LOS and OLOS segments are discussed, respectively, as well. The results reveal that angle spread of scattering component in OLOS is a little bigger than that in LOS segment. And AOA of scattering component center on 90 degrees approximately following a Gaussian distribution in OLOS segment due to the scatterers near to the receiver; it varies around $\widehat{\theta}_{\mathrm{LOS}}$ in LOS segment because of the scatterers near the transmitter. The statistical probability of $\eta$ in the two segments is analysed in brief finally. A new parametric multipath fading model is proposed. The closed form correlation function and Doppler spectrum in a tree-blocked HSR viaduct scenario are given. The parameters related to angle scattering are estimated and the statistical models of the channel parameters are established. The channel model proposed in this paper would better describe the real viaduct scenario and could be used for evaluation of the communication system simulations. 


\section{Conflict of Interests}

The authors declare that there is no conflict of interests regarding the publication of this paper.

\section{Acknowledgments}

The research was supported in part by the NSFC Project under Grants nos. 61371070 and 61032002, the Open Research Fund of National Mobile Communications Research Laboratory, Southeast University (no. 2012D07), and the Fundamental Research Funds for the Central Universities under Grant 2013 YJS016.

\section{References}

[1] Wikipedia, "High-speed rail," 2011, http://en.wikipedia.org/wiki/ High-speed_rail.

[2] Wikipedia, "High-speed rail," 2011, http://en.wikipedia.org/wiki/ Eurostar.

[3] Wikipedia, "High-speed rail," 2011, http://en.wikipedia.org/wiki /High-speed_rail_in_China.

[4] J. Li and M. Kavehrad, "Effects of time selective multipath fading on OFDM systems for broadband mobile applications," IEEE Communications Letters, vol. 3, no. 12, pp. 332-334, 1999.

[5] L. Liu, C. Tao, J. Qiu et al., "Position-based modeling for wireless channel on high-speed railway under a viaduct at $2.35 \mathrm{GHz}$," IEEE Journal on Selected Areas in Communications, vol. 30, no. 4, pp. 834-845, 2012.

[6] 3GPP, TS 36. 104 V9. 3. 0 3rd Generation Partnership Project, Technical Specification Group Radio Access Network, Evolved Universal Terrestrial Radio Access (e-utra), Base Station (BS) radio transmission and reception (Release 9), 2010.

[7] R. Parviainen and K. Pekka, "Results of high speed train channel measurements," in European Cooperation in the Field of Scientific and Technical Research, 2008.

[8] J. Dulmage and M. P. Fitz, "Nonisotropic fading channel model for the highway environment," IEEE Vehicular Technology Magazine, vol. 2, no. 4, pp. 12-18, 2007.

[9] A. Abdi, J. A. Barger, and M. Kaveh, "A parametric model for the distribution of the angle of arrival and the associated correlation function and power spectrum at the mobile station," IEEE Transactions on Vehicular Technology, vol. 51, no. 3, pp. 425-434, 2002.

[10] "Sencity Rail Antenna: 1399. 17. 0039 HUBER+SUHNER data sheet," HUBER+SUHNER AG RF Industrial, 2010.

[11] Q. Spencer, M. Rice, B. Jeffs, and M. Jensen, "Statistical model for angle of arrival in indoor multipath propagation," in Proceedings of the 47th IEEE Vehicular Technology Conference, vol. 3, pp. 1415-1419, May 1997.

[12] M. D. Austin and G. L. Stuber, "Velocity adaptive handoff algorithms for microcellular systems," IEEE Transactions on Vehicular Technology, vol. 43, no. 3, pp. 549-561, 1994.

[13] R. Gonin, Nonlinear Lp-Norm Estimation, vol. 100, CRC Press, 1989. 

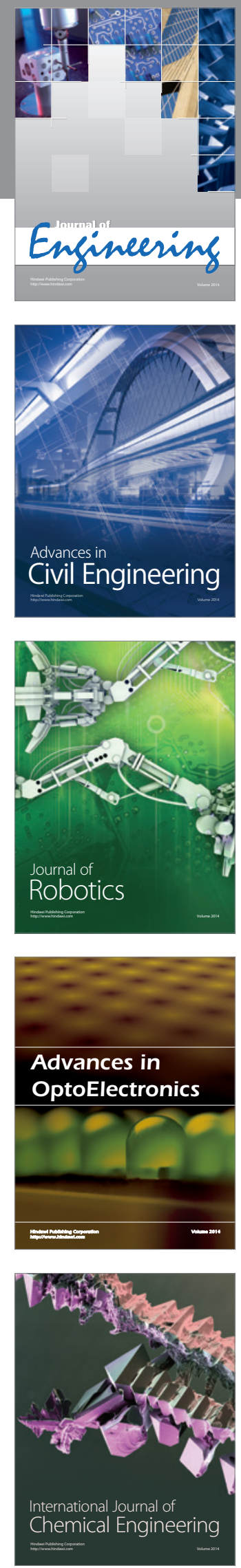

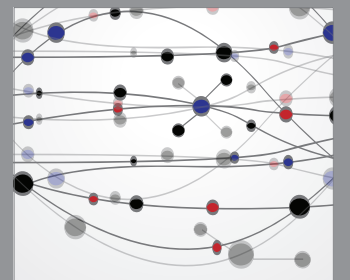

The Scientific World Journal
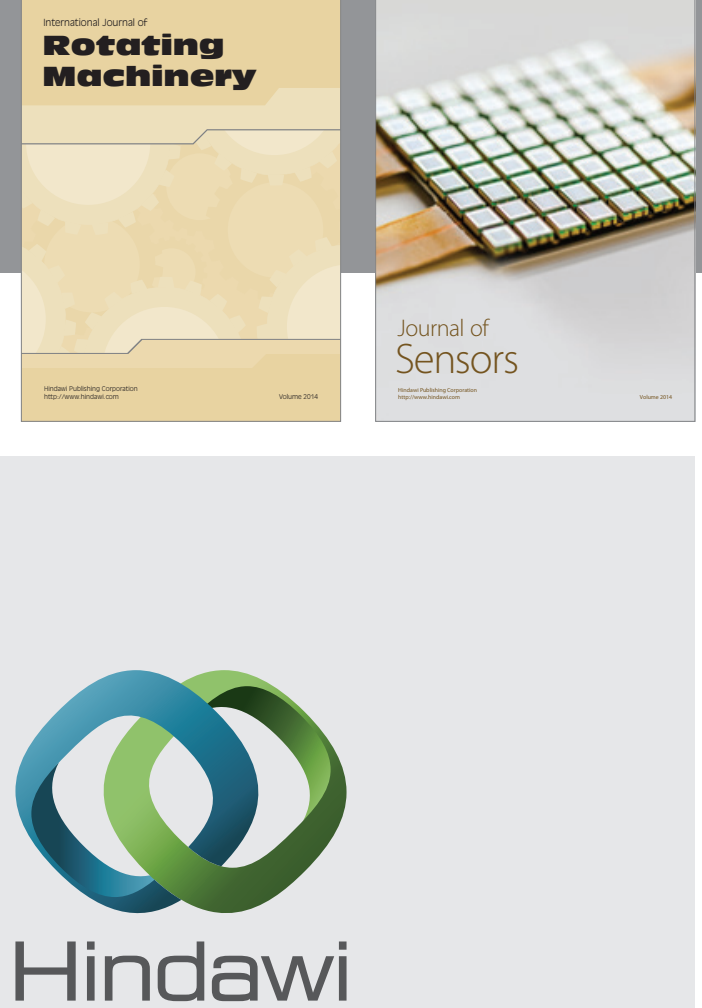

Submit your manuscripts at http://www.hindawi.com
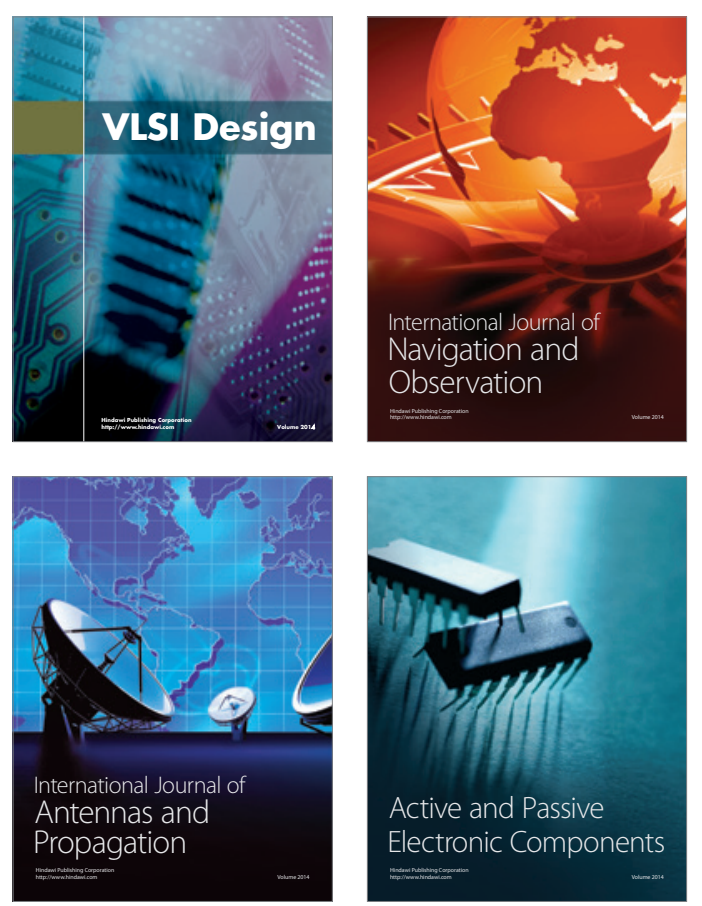
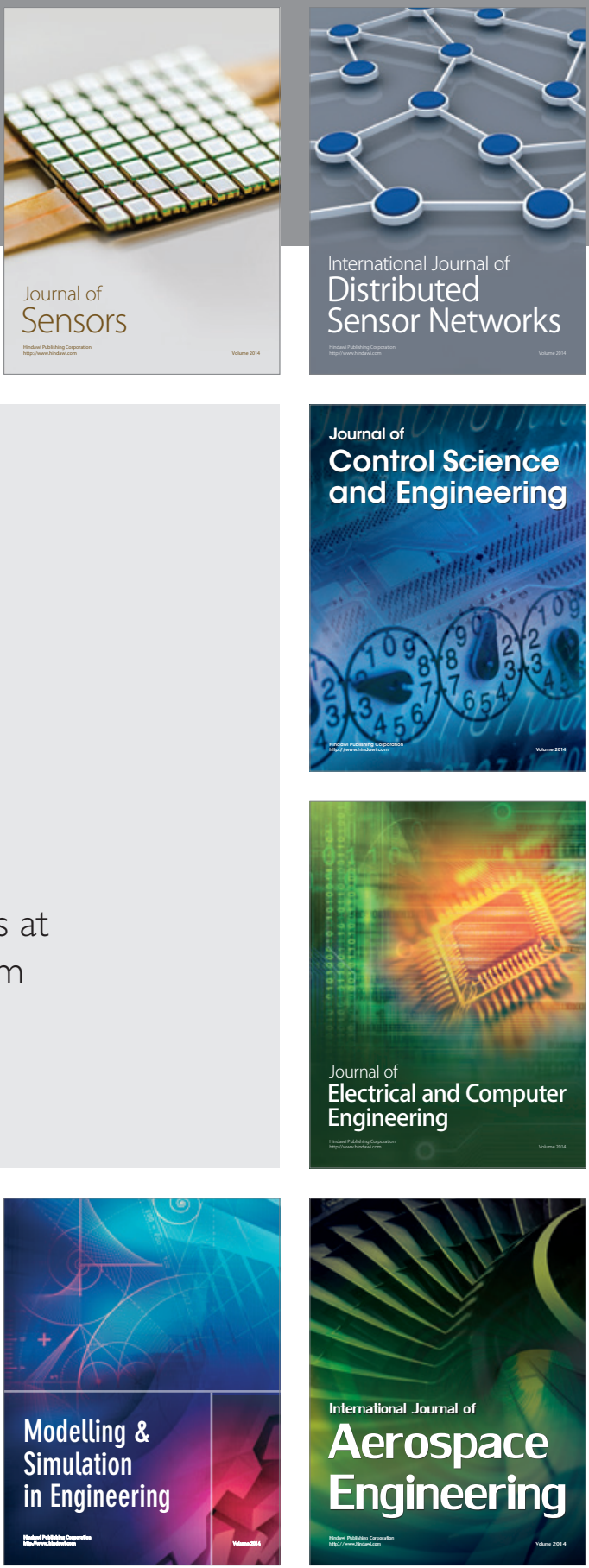

Journal of

Control Science

and Engineering
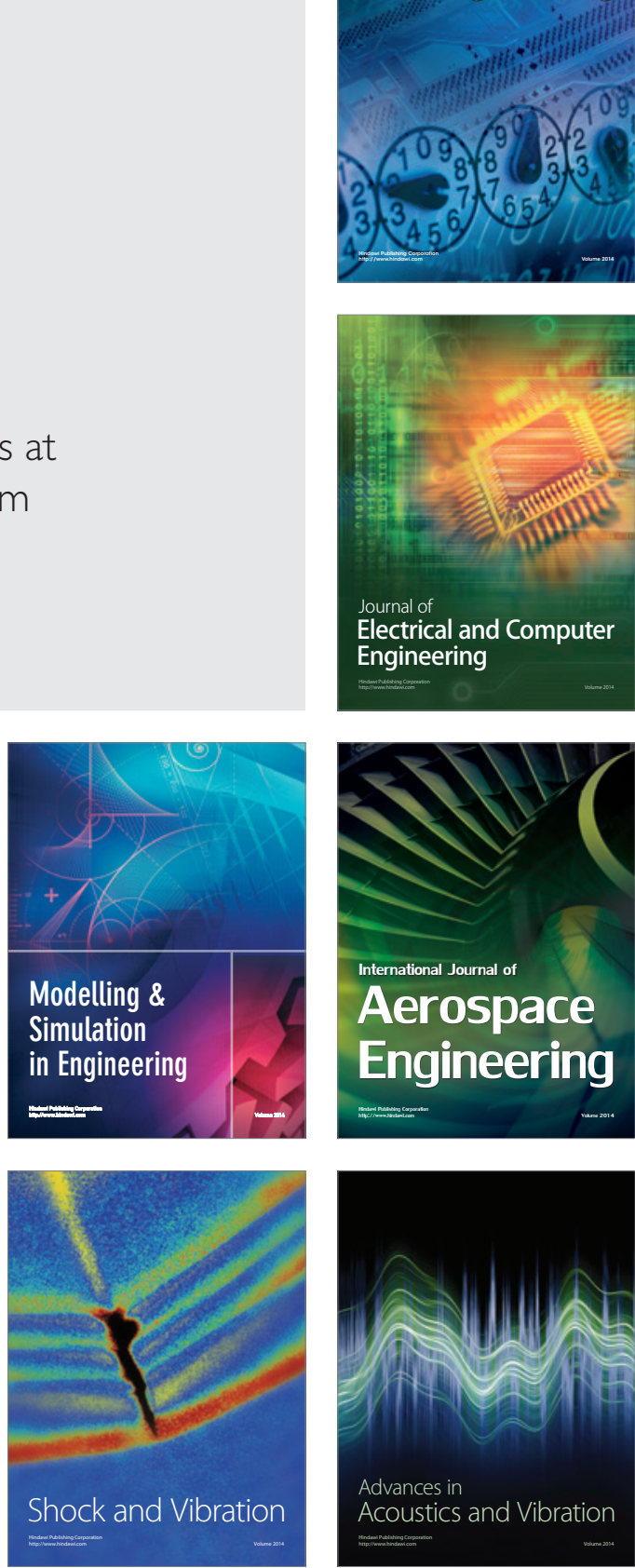